\title{
Unbotmäßige Literaturgeschichtsschreibung deutsch. Friedrich Schlegels Wiener Vorlesungen Geschichte der alten und neuen Literatur (1812)
}

Bereits vier Jahre, nachdem Friedrich Schlegel seine Wiener Vorlesungen Geschichte der alten und neuen Literatur 1814 in den Druck gegeben hatte, ${ }^{1}$ erschien in Edinburgh ihre englische Übersetzung. ${ }^{2}$ Unter dem Titel Lectures on the History of Literature, Ancient and Modern erfuhr diese Übersetzung in den folgenden Jahrzehnten zahlreiche Neuauflagen und -ausgaben, ${ }^{3}$ im Jahre 1841 auch die erste US-amerikanische: „We may be allowed to add“, so schließt der New Yorker Herausgeber seine Vorrede, „that we possess few works, not written in our own vernacular, which discover the ornaments of a more polished rhetoric, or one characterized by greater felicity of expression and style“, was zwar nicht zuletzt dem begnadeten Übersetzer zuzurechnen sei, doch im selben Maße eben auch ,the genius of the original, and the rich exuberance of German idiom“. ${ }^{4}$ Dass es sich bei Schlegels Literaturgeschichtsvorlesungen um ein „work of permanent value“ handelt, steht für den amerikanischen Editor außer Frage. Entsprechend versieht er seinen Neudruck der Edinburgher Übertragung zusätzlich mit einem Namens- und Schlagwortregister, „with the view of facilitating reference, and rendering it more complete as a standard synoptical book on all subjects connected with our literary annals“. 5

Es ist nicht ohne Signifikanz, dass es einer Vermittlung durch die englischsprachige Publikations- und Rezeptionsgeschichte bedarf, um in Deutschland überhaupt auf die Idee zu kommen, Friedrich Schlegels Geschichte der alten

1 Die Vorlesungen sind, datiert auf das Folgejahr, 1814 erschienen: Schlegel 1815.

2 Schlegel 1818. 1819 folgte eine zweite Auflage.

3 Auf Grundlage der Daten des Karlsruher Virtuellen Katalogs habe ich bis in die 1880er Jahre zehn Ausgaben im Vereinigten Königreich gezählt, vgl. https://kvk.bibliothek.kit.edu (19.07.2019). Eine Forschung zur Rezeption der Vorlesungen im englischsprachigen Raum existiert nicht.

4 Schlegel 1841b: vi f. Die Übersetzung stammt von John Gibson Lockhart, Esq. aus dem Jahre 1818 .

5 Ebd.: vii. Die Londoner Ausgabe von 1859 publiziert die Vorlesungen dann ebenfalls mit einem Register: Schlegel 1859.

○ Open Access. (c) 2019 Andrea Polaschegg, publiziert von De Gruyter. (c) BY-NC-ND Dieses Werk ist lizenziert unter der Creative Commons Attribution-NonCommercial-NoDerivatives 4.0 Lizenz.

https://doi.org/10.1515/9783110676631-006 
und neuen Literatur als ein literaturhistorisches Grundlagenwerk und zugleich als ein Eckdatum der deutschen Wissenschaftssprache zu begreifen. Wie ich im Folgenden darlegen will, schreibt sich dieser doppelte Statuszuweis an Schlegels Vorlesungen nämlich keineswegs aus einem wie auch immer gearteten Spleen unserer anglophonen Nachbarn her. Vielmehr resultiert umgekehrt die anhaltende und teils programmatische Dekanonisierung der Geschichte der alten und neuen Literatur hierzulande ${ }^{6}$ aus einer sehr deutschen und überdies sehr nationalphilologischen Idiosynkrasie gegenüber diesem traditionell ungelesenen Text, seinem Autor und seinem konkreten Entstehungskontext.

Eine eingehendere Beschäftigung mit Schlegels Wiener Literaturgeschichtsvorlesungen verspricht somit nicht allein Aufschluss über die Entwicklungsgeschichte des Deutschen als Wissenschaftssprache, sondern auch über die weltanschauliche Grundierung der gegenwärtigen Literaturwissenschaft samt ihrer disziplinären und politischen Traditionslinien. Beides kulminiert, soviel sei vorweggenommen, in der zentralen Bezugsgröße der ,Nation(alliteratur)“, deren staatspolitische und konfessionskulturelle Implikationen das Gegenstandsverständnis der heutigen Germanistik in Deutschland nicht weniger stark prägen, als sie diejenige Öffentlichkeit bestimmt haben, der Friedrich Schlegel in den 1810er Jahren seine Vorlesungen präsentierte - wenngleich unter umgekehrtem Vorzeichen.

\section{Erinnerung und Bewusstsein: performativ}

Als Vortragender war Friedrich Schlegel in Wien kein Unbekannter mehr, als er vom 27. Februar bis zum 30. April 1812 im Tanz- und Konzertsaal des renommierten Gasthofs „Zum römischen Kaiser“ auf der Freyung seine insgesamt 16 literaturgeschichtlichen Vorlesungen hielt. Schon im Frühjahr 1810 hatte er öffentlich Über die neuere Geschichte gelesen, was in der Wiener Gesellschaft großen Anklang fand, und diese Vorlesungen im Folgejahr schließlich „ganz so, wie sie gehalten wurden“, mit enormer Resonanz publiziert. ${ }^{7}$ So kann es nicht

6 Nach der Aufnahme der Vorlesungen in die von Schlegel selbst besorgte Werkausgabe (1822-1846), wo sie bezeichnenderweise die ersten beiden Bände füllen, hat die Geschichte der alten und neuen Literatur in Deutschland bis heute nur zwei weitere Ausgaben mit je einer Auflage erfahren: Schlegel 1841a und Schlegel 1911.

7 Schlegel 1811: Vorrede, o.P. Umgekehrt proportional zum, im Übrigen auch internationalen, Erfolg dieser Wiener Geschichtsvorlesungen verhält sich die Forschung zu den Texten. Das Friedrich Schlegel Handbuch widmet ihnen eine magere Spalte im Segment „Werke und Werkgruppen“ ohne eigenes Lemma (Schöning 2017: 250f.) sowie eine gute halbe Spalte im biogra- 
verwundern, dass auch Schlegels Vortrag seiner Geschichte der alten und neuen Literatur zu einem gesellschaftlichen Ereignis wurde und rund 200 überwiegend adelige Wienerinnen und Wiener ${ }^{8}$ Karten erwarben, um sich - wie es in der Ankündigung der Veranstaltung im Oesterreichischen Beobachter vom 19. Februar $1812 \mathrm{zu}$ lesen stand - „,[d]ie Litteratur in ihrem vielfachen Einflusse auf das wirkliche Leben, auf das Schicksal der Nationen und den Gang der Zeiten dar[ ]stellen“ zu lassen. ${ }^{9}$ Der junge Joseph von Eichendorff war ebenfalls dabei und notierte zur ersten Vorlesung in sein Tagebuch: „Großes Publikum. Vorn Kreis von Damen, Fürstin Liechtenstein mit ihren Prinzessinnen, Lichnowsky, etc. 29 Fürsten. Unten großes Gedränge von Equipagen, wie auf einem Ball. Sehr brillant.“10

Wie kurz nach ihm auch Adam Müller zielte Schlegel mit seinen Wiener Vorlesungen auf ein gesellschaftlich und politisch einflussreiches Publikum außerhalb der institutionalisierten Gelehrsamkeit und ihrer Räume $a b^{11}$ und folgte damit auf der Ebene der rhetorischen actio demselben Kalkül wie in der Behandlung und Darstellung seines literarischen Gegenstandes. Er habe es sich zur Aufgabe gemacht, so heißt es in der Widmungsvorrede der Druckfassung an den Fürsten von Metternich 1814 noch einmal pointiert, mit seiner Geschichte der alten und neuen Literatur „der großen Kluft, welche immer noch die literarische Welt und das intellektuelle Leben des Menschen von der praktischen Wirklichkeit trennt, entgegen zu wirken“, und zwar durch „das darin aufgestellte Gemälde von der Geistesbildung der merkwürdigsten Völker Europas“. ${ }^{12}$

Anders als die Vollmundigkeit dieses Anspruchs und die holistische Formulierung des Vorlesungstitels vermuten lassen könnten, löst Friedrich Schlegel sein Versprechen, in der Geschichte der alten und neuen Literatur „den Geist der Literatur in jedem Zeitalter, das Ganze derselben, und den Gang ihrer Entwicklung bei

phischen Segment (Breuer 2017), deren Darstellung auf die immerhin ein wenig umfangreicheren Ausführungen in Ernst Behlers Biographie zurückgeht, vgl. Behler 1996: 112-117.

8 Zum Publikum vgl. die Einleitung von Hans Eichner in der Kritischen Friedrich-SchlegelAusgabe: Eichner 1961: XXIII.

9 O.A. 1812.

10 Eichendorff 1980: 700.

11 Zur Diskurspolitik und Gattungspoetik von Müllers Vorlesungen (Müller 1967) vgl. den differenzierten Beitrag von Peter Schnyder in diesem Band. Die kulturpolitischen Strategien von Müllers und Schlegels Wiener Vorlesungen vergleichend herausgearbeitet hat Herrmann 2015: 227-248.

12 Schlegel 1961: 4. Nachweise hieraus im Folgenden mit Angabe der Seitenzahl direkt im Text. Zur Bedeutung dieses Programms eines gestaltenden Einflusses der Literatur auf das „Leben“ bei Schlegel vgl. Marquardt 1995: $28 \mathrm{f}$. 
den wichtigsten Nationen“ (6) darzustellen, tatsächlich ein: Die 16 Vorlesungen enthalten nicht weniger als die erste gesamteuropäische Literaturgeschichte, die von der Antike bis in die Schlegel'sche Gegenwart reicht und über die Einbeziehung der orientalischen Literaturgeschichte in einen welthistorischen Horizont eingestellt wird, wobei unter ,Literatur` keineswegs allein Dichtung, sondern gleichermaßen Philosophie, Historiographie und Rhetorik - also die litterae in ihrer Gesamtheit - gefasst und in ihrer Wechselwirkung mit dem „Schicksal der Nationen und de[m] Gang der Zeiten“ (9) sichtbar gemacht werden.

Für das Verständnis sowohl der historiographischen Konzeption und induktiven Epistemologie als auch der politischen und kulturellen Wirkungsabsicht der Vorlesungen ${ }^{13}$ besonders entscheidend ist der weite Literaturbegriff, der ihnen zugrunde liegt, zusammen mit dem Umstand, dass Schlegel seine europäische Literaturgeschichte welthistorischer Abmessung eben in deutscher Sprache vorgetragen und publiziert hat, ${ }^{14}$ und dies beides in Wien. Wenn seine Vorlesungen, so heißt es in der Vorrede zur Druckfassung, unter „Literatur“ weit mehr verhandelten, „als man sonst wohl unter jener Überschrift zu erwarten gewohnt ist“, so sei dies weder „Auswuchs“ noch Frucht des Zufalls, sondern es resultiere aus dem „in diesem Werke durchgehends herrschenden Begriff von Literatur, als dem Inbegriff des intellektuellen Lebens einer Nation“ (7).

Nur unter dieser Voraussetzung gewinnt das Projekt einer Literaturgeschichtsschreibung für Schlegel überhaupt Relevanz, und ihr verdankt die Geschichte der alten und neuen Literatur auch ihre genuin politische Pointe, die in der eigentümlich rekursiven Bewegung der Vorlesungen liegt:

Die zentrale Ordnungskategorie der Schlegel'schen Weltliteraturgeschichtsschreibung - Jürgen Fohrmann nennt sie ihre „klassifikatorische Dominante“15 _ ist, wie bereits erwähnt, die Nation. Als konstitutiv für die Entwicklung jeglicher Nation behauptet Schlegel nun einerseits die Existenz „große[r] NationalErinnerungen“ eines Volkes (15), für deren Bewahrung und Tradierung die Dichtkunst zuständig ist (59), zum anderen das Vorhandensein eines „klaren Bewußtsein[s] seiner eigenen Taten und Schicksale“ (16) in Form der Ge-

13 Eine ausführliche Darstellung dieser Konzeption und der Strategien, nach denen Schlegel sein immenses Material zu einer geschlossenen historiographischen Erzählung ordnet, findet sich in Polaschegg 2017: 224-233.

14 Nicht zuletzt in dieser Spannung zwischen europäischem Gegenstand und deutscher Sprache unterscheiden sich Schlegels Literaturgeschichtsvorlesungen nicht nur kategorial von denen Adam Müllers, sondern auch von den nachfolgenden deutschsprachigen Literaturgeschichten, namentlich von Georg Gottfried Gervinus' Geschichte der poetischen NationalLiteratur der Deutschen (5 Bde. Leipzig 1835-1842) und Joseph von Eichendorffs Geschichte der poetischen Literatur Deutschlands (2 Bde. Paderborn 1857).

15 Fohrmann 1989: 111. 
schichtsschreibung. Ohne Nationalerinnerungen im Speicher der Dichtung und ohne Geschichtsschreibung als reflektierende Produktionsinstanz von Selbstbewusstsein im Wortsinne können Völker, dieser Logik folgend, keine Nationen werden.

Mit seiner Geschichte der alten und neuen Literatur reiht Schlegel sich selbst also zunächst performativ in eben diejenige Literaturgeschichte ein, die seine Vorlesungen als Literatur allererst schreiben. ${ }^{16}$ Und weil es sich dabei um Geschichtsschreibung handelt, produziert der Verfasser zugleich und ebenfalls performativ historiographisches Selbstbewusstsein und arbeitet somit tatsächlich ganz praktisch an der Konstitution einer deutschen Nation mit. ${ }^{17}$

Als erste Literaturgeschichte in deutscher Sprache sind Schlegels Wiener Vorlesungen also per se bereits ein nationalpolitisches Projekt, und dies gerade nicht aufgrund ihres Gegenstandsbereichs, der ein ausgewiesen transnationaler ist, sondern wegen ihres historiographischen Formats und ihres Charakters als deutschsprachige Literatur.

\section{Literaturgeschichte in der „preußisch- kleindeutschen Schule“}

Seine besondere Kontur gewinnt dieses Projekt - und gewinnt auch dessen Diskussion im Rahmen einer Auseinandersetzung mit dem Deutschen als Wissenschaftssprache - allerdings dadurch, dass Friedrich Schlegel seine Vorlesungen eben in Wien hält und sie auch dort publiziert. Der Zusammenhang von deutscher Sprache, Literatur und Nation präsentiert sich aus der Perspektive der Hauptstadt des Habsburger Vielvölkerreichs im Jahre 1812 nämlich als ein ungleich spannungsreicherer und auch ungleich spannenderer als in einem Horizont, der auf den Raum um Weimar, Leipzig, Göttingen, Frankfurt oder Berlin beschränkt ist: Im Herrschaftsgebiet des seit 1804 bestehenden österreichischen Kaisertums (dem nach Russland zweitgrößten Staat Europas) war das Deutsche - trotz der entsprechenden Reformversuche Josephs II. - weder Schulsprache noch Amts-

16 Auf den performativen Charakter der Vorlesungen hat auch Christopher Busch hingewiesen, der darin eine „Reminiszenz frühromantischer Versuche“ sieht, „den Prozesscharakter der Dichtung reflexiv zu beschreiben“ (Busch 2011: 38).

17 Zwar gebe ich Britta Herrmann recht, wenn sie auf den wichtigen Beitrag von Schlegels Zeitschriften - darunter auch das ebenfalls 1812 in Wien gegründete Deutsche Museum - für den Brückenschlag zwischen Literatur und Leben im Zeichen der Nation hinweist (vgl. Herrmann 2015: 229, 235f.), doch das Potential der historiographischen Darstellungsform als Generator nationalen Selbstbwusstseins geht noch weit darüber hinaus. 
sprache, und aufgrund der Dominanz des Katholizismus in den Kronländern spielte es auch in der religiösen Kultur und Praxis nur eine untergeordnete Rolle. Stattdessen diente das Lateinische in diesem Vielvölkerreich als eine lingua franca und erfüllte wichtige staats- und gesellschaftskulturelle Integrationsfunktionen. Eine deutschsprachige Dichtung eigener poetischer Dignität begann sich hier entsprechend erst Mitte des 18. Jahrhunderts langsam zu formieren und sich noch langsamer gegen die lateinische Dichtung durchzusetzen. ${ }^{18}$

Welches Textkorpus zu welcher Zeit eine Geschichte der deutschsprachigen litterae eigentlich umfasst und vor allem, welche konkreten Abmessungen die Nation besitzt oder besitzen soll, die diese Literatur zu einer ,Nationalliteratur macht und vice versa, ist mit Blick auf die deutschsprachigen Teile Habsburgs also eine durchaus neuralgische Frage - und dies nicht allein für Friedrich Schlegel und auch nicht allein während der Jahrzehnte um 1800.

Auf die weitreichenden Differenzen zwischen Wien als Hauptstadt eines Kaiserreichs, dessen Grenzen jede wie auch immer zugeschnittene deutsche Nation weit überstiegen, und den deutschen Staaten(bünden), die allesamt kleiner waren als eine solche Nation, eigens hinzuweisen, erscheint mit Blick auf die etablierten Parameter germanistischer Forschung und Historiographie alles andere als obsolet. Schließlich tendiert die deutsche Literatur- und Kulturwissenschaft traditionell dazu, selbst in der historischen Rückschau terminologisch und konzeptuell das ,Deutschsprachige“ mit dem ,Deutschen' und beides derart selbstverständlich mit ,Deutschland' als Referenzgröße der ,Nationalliteratur‘ kurzzuschließen, als hätte es Habsburg bzw. Österreich und die Schweiz als eigenständige staatspolitische und gesellschaftliche Größen nie gegeben - oder zumindest nicht in literatur- und kulturgeschichtlich relevanter Weise. Aller diskurs- und systemtheoretischen Schulung zum Trotz wird österreichische und schweizerische Literatur, wenn sie sich (wie etwa im Falle des realistischen Romans und der klassischen Moderne) als kanonkonstitutiv präsentiert, von der deutschen Literaturwissenschaft jedenfalls durchweg wie deutsche Literatur behandelt oder, wo dies nicht der Fall ist, ausgeblendet.

Michael Böhler hat diese bemerkenswerte Fachtendenz zur wechselseitigen Überblendung des ,Deutschsprachigen“ mit dem ,Deutschen“ in seiner erhellenden Analyse germanistischer Literaturgeschichten seit der Nachkriegszeit auf den schönen Begriff des „synekdochische[n] Glissando[s]“ gebracht ${ }^{19}$ und auf die starken kulturhegemonialen Kräfte hingewiesen, die dabei wirken. Ein wenig überpointiert könnte man somit konstatieren, dass die literatur- und kultur-

18 Vgl. den Überblick in Zeyringer 2012: 45-126; differenziert: Michler 2011: 187-206.

19 Böhler 2002: insb. 20-23. 
wissenschaftliche Fachpraxis in Deutschland mit ihrem synekdochischen Konzept des ,Deutsch(sprachig)en“ sich insgesamt im Rahmen einer „preußischkleindeutschen Schule“ bewegt. Unter dieser Rubrik hatten Friedrich Jaeger und Jörn Rüsen in ihrer Geschichte des Historismus Historiker wie Mommsen, Droysen, Treischke und Gervinus zusammengefasst und von anderen historiographischen Ansätzen des 19. Jahrhunderts - etwa denen Rankes oder Niebuhrs - abgegrenzt, deren Horizont Habsburg und die Schweiz selbstverständlich mit umfasste. ${ }^{20}$

Wenn Friedrich Schlegel 1812 in Wien bei seinem Projekt einer nationalkonstituierenden Literaturgeschichtsschreibung europäischer Provenienz die deutsche Sprache performativ zum alles entscheidenden Medium wählt, dann reagiert er damit auf eben jenes zutiefst ambivalente Verhältnis von Sprache, Nation, Gesellschaft und Staat, das die deutschsprachige Literatur von jeher geprägt hat und bis heute prägt, während es die deutsche Literaturwissenschaft durch ihr „Synekdochisches Glissando“ zwischen dem ,Deutschsprachigen“ und dem ,Deutschen" traditionell blind macht.

Dass diese literaturwissenschaftliche Blindheit tatsächlich eine „preußischkleindeutsche“ Prägung besitzt und durchaus kulturhegemoniale Züge trägt, lässt sich an der Rezeption der Geschichte der alten und neuen Literatur gut ablesen, die innerhalb des Fachs bekanntlich kaum stattgefunden hat, obwohl an der exzeptionellen Bedeutung des Werks für die Literaturgeschichtsschreibung kein Zweifel bestehen kann. ${ }^{21}$ Besonders augenfällig wird dies am konfessionspolitischen Kalkül, dem die Dekanonisierung von Schlegels Literaturgeschichte in guter preußischer Tradition folgt, insofern mit der Konversion des Autors zum Katholizismus im Jahr 1808 eine kategoriale werkgeschichtliche Zäsur gesetzt wird, die das ,progressive‘, ,kosmopolitische‘ und ,liberale‘ Frühwerk vom ,restaurativen‘, ,patriotisch-nationalistischen“ und ,reaktionären“ Spätwerk trennt. ${ }^{22}$ „Anstößig wirkt“, so formuliert es Stefan Matuschek stellvertretend für viele andere deutsche Fachvertreter, „der Katholizismus seiner späten Jahre, der das am jüngeren Schlegel bewunderte kritische Urteilsvermögen und die historische Kenntnisweite zu einseitiger, extremer Religiosität verschiebt. Der Pionier kritischer historischer Wissenschaft wird zum konfessionellen Eiferer."23 Zusammen mit Wien als ihrem Ereignisraum und Metternich als Adressat ihrer Widmungsvorrede fundiert dies die weithin geteilte Einschätzung, die Geschichte der alten und neuen Literatur sei

20 Jaeger \& Rüsen 1992: 86-92.

21 Darauf hatte bereits René Wellek hingewiesen, vgl. Wellek 1978: 281-293; ebenso Marquart 1995: 27-31 und Fohrmann 1989: 110-114.

22 Vgl. dazu exemplarisch Bauer 2014: 161; Breuer 2010: 69.

23 Matuschek 2001: 194. 
„eines der Gründungsdokumente wissenschaftlicher Literaturgeschichtsschreibung und zugleich ein Dokument katholisch restaurativer Tendenz“, ${ }^{24}$ was die Vorlesungen an den Rand des literaturwissenschaftlichen Lektürekanons verschoben hat, wo nicht darüber hinaus.

Die Mechanismen dieser bis heute erfolgreichen programmatischen Ignoranz gegenüber Schlegels Vorlesungen vor dem - sei es explizierten, sei es implizit bleibenden - Hintergrund seiner Konversion kann man im Übrigen am literaturwissenschaftlichen Umgang mit den Werken sämtlicher konvertierter Autorinnen und Autoren der deutschsprachigen Literatur verfolgen. Angefangen von Adam Müller, Clemens Brentano und Zacharias Werner über Hermann Bahr, Hugo Ball und Gertrud von le Fort bis hin zu Alfred Döblin: überall markiert der Übertritt der Autorinnen und Autoren zum Katholizismus die Grenze ihres literaturwissenschaftlich wahr- und ernstgenommenen Werks. ${ }^{25}$ Die besondere Pointe liegt im Falle der Geschichte der alten und neuen Literatur indes darin, dass der literaturwissenschaftliche Kurzschluss von Schlegels Konversion mit dem Verlust „kritischer Urteilskraft“ und „historischer Kenntnisweite“26 ausgerechnet die kulturräumlich und -zeitlich mit Abstand umfänglichste Literaturgeschichte des gesamten 19. Jahrhunderts betrifft. Und wie im Folgenden zu zeigen sein wird, liegt in der tatsächlich katholischen Justierung der Schlegel'schen Vorlesungen zugleich die Bedingung der Möglichkeit ihres europäischen Ausgriffs im weltliterarischen Horizont, während Wien den konstitutiven Bezugspunkt für die performative Schaffung einer Nation in den Abmessungen des deutschsprachigen Raums bildet.

Dies sei nun genauer nachgezeichnet, wobei die Darstellung angesichts der ungeheuerlichen Menge an Sprachen, Texten und Zusammenhängen, die Schlegel auf den rund 400 Seiten seiner Vorlesungen durcharbeitet und historiographisch arrangiert, notwendig skizzenhaft bleiben muss. ${ }^{27}$

24 Ebd.

25 Die Geschichte dieser konfessionsgrundierten Dekanonisierungspolitik, ihrer nationalen Hintergründe und Folgen ist noch nicht geschrieben. Ansätze finden sich bei Osinski 1993; auf die Spur des Protestantismus der deutschen Literaturwissenschaft und seiner epistemologischen und politischen Effekte hat sich zuletzt Clemens Pornschlegel gesetzt: Pornschlegel 2017.

26 Vgl. dazu kritisch Wegmann 2017: insb. $156 \mathrm{f}$.

27 Vgl. dazu noch einmal ausführlicher Polaschegg 2017; Polaschegg 2019. 


\section{Sprache, Nation und Literatur: Ordnungsversuche}

Das zentrale Medium sowohl der Schlegel'schen Vorlesungen selbst als auch ihres Gegenstandes ist die „Muttersprache“ (9-11): Allein in ihr hätten sich die Nationalerinnerungen der Völker artikuliert, von denen die höfische Gesellschaft durch ihre Frankophonie und der Gelehrtenstand durch das Lateinische ebenso abgekoppelt gewesen seien wie voneinander getrennt. „Die Trennung des gelehrten Standes und der gesellschaftlichen Bildung unter sich und von dem Volke“, so hält Schlegel gleich zu Beginn der ersten Vorlesung programmatisch fest, „ist das größte Hindernis einer allgemeinen Nationalbildung“ (11). Denn hier gelte der Grundsatz: „[U]nzertrennlich ist Geist und Sprache, [...] wesentlich eins Gedanke und Wort“(14). Seit Mitte des 18. Jahrhunderts aber und auf diese Datierung wird zurückzukommen sein - sei die Wertschätzung der deutschen Sprache und der in ihr verfassten Werke deutlich gestiegen, was nicht nur die Hoffnung auf eine ständeübergreifende Einheit nähre, sondern auch auf die Rückbesinnung der Gelehrten und Gebildeten auf die besagten Nationalerinnerungen (10).

An eben diesem Punkt setzt das performative Kalkül von Schlegels deutschsprachiger Literaturgeschichte an, die ihrem Selbstverständnis nach als gesellschaftliches Integral und als Brückenschlag zu den „vaterländischen Erinnerungen“ (10) gleichermaßen dienen soll. Angesichts dieser starken Akzentuierung der Muttersprache ist es umso bemerkenswerter, dass der Verfasser die An- und Zuordnungen der europäischen und orientalischen Nationalliteraturen gerade nicht über die Verwandtschaftsbeziehungen ihrer Sprachen organisiert. Schließlich hatte Friedrich Schlegel nicht einmal sechs Jahre zuvor seine für die Entwicklung der vergleichenden Sprachwissenschaft so richtungsweisende Schrift Über die Sprache und Weisheit der Indier ${ }^{28}$ vorgelegt und darin die bahnbrechende Erkenntnis formuliert, dass sich die Weltsprachen nach ihren jeweiligen Bildungsgesetzen zu verschiedenen Sprachfamilien gruppieren lassen, die untereinander wiederum keinerlei Verwandtschaft aufweisen: ${ }^{29}$ Die germanischen Sprachen bildeten zusammen mit dem Sanskrit, dem Persischen, dem Griechischen und dem Lateinischen die indogermanische Sprachfamilie, der die nicht flektierenden Sprachen - allem voran das Chinesische - diametral gegenüberstanden und die auch

28 Schlegel 1975.

29 Zur Einordnung Friedrich Schlegels in die Geschichte der vergleichenden Sprachwissenschaft sowie der Frühindologie vgl. nach wie vor einschlägig Windisch 1992: 55-86. 
von der semitischen Sprachfamilie mit dem Hebräischen und dem Arabischen kategorial unterschieden wurde. ${ }^{30}$

In Schlegels Literaturgeschichte dagegen finden die Sprachfamilien weder Erwähnung noch kompositorischen Niederschlag. Stattdessen stellt er die sprach- und kulturgeschichtlich vergleichsweise junge - griechische Literatur an den Anfang seiner historiographischen Erzählung, gefolgt von der römischen, um beide dann mit der hebräischen zu flankieren, was nach den Gesetzen der Sprachverwandtschaft unmöglich wäre.

Die Initialstellung der Griechen begründet der Verfasser damit, dass sich deren „Geistesbildung [...] ganz aus sich selbst entwickelt“ habe „und fast ganz unabhängig von der Bildung anderer Nationen entstanden“ sei (19). Solche Formen kultureller Autarkie gelten Schlegel im Laufe seiner Vorlesungen immer wieder als besonderer Qualitätsausweis einzelner Nationalliteraturen, deren Niedergang umgekehrt mit der Übernahme fremder Gestaltungsprinzipien in Verbindung gebracht wird. Entsprechend prekär ist der Stand der römischen Literatur, die sich durch übermäßige „Nachkünstelung“ (66) griechischer Gattungen und Formen ausgezeichnet und daher nie einen wirklichen „Originalgeist“ entwickelt habe (66-68), abgesehen allerdings von einem für Schlegel wesentlichen Punkt: der Herausbildung der großen „Idee von Rom“ (69) als zentraler Referenzgröße von Literatur, Staat und Gesellschaft. Auf diese vaterländische Idee sei sowohl die einzige originäre Gattung der Römer, die dezidiert urbane Satire, bezogen (82) als auch die Aeneis Virgils als „eigentliche[s] Nationalgedicht der Römer“ (80). Mit dem Verlust der Stadt Rom als staatspolitischem und kulturellem Zentrum sowie als Inbegriff der patria habe, so Schlegel, dann auch der Verfall der römischen Literatur begonnen. Es ist also das kaiserliche Rom, das die Blüte seiner Literatur garantierte, während das republikanische ihren Niedergang einläutete.

Analog dazu hatte Schlegel bereits die griechische Literatur epochalisiert, an deren Anfang er Homer als „Bürge und Zeuge der alten Heldensage und Heldenzeit“ (28) stellt, nicht ohne den königlichen Status sämtlicher Figuren der Ilias zu betonen. Auf den solcherart als Epiker und Historiker vorgestellten Homer folgen Pindar, Aischylos und Herodot, die eng mit den Perserkriegen verbunden werden, während nach Sophokles bereits der Verfall der griechischen Literatur einsetzt. Sophismus und Republikanismus sind die Faktoren, die Schlegel dafür verantwortlich macht und denen er auch das Erbe Platons und Aristoteles' zum Opfer fallen sieht, um zugleich Aristophanes als genialen literarischen Kommentator dieses Niedergangs zu würdigen (42-44).

30 Vgl. dazu noch einmal ausführlich Endres 2017. 
Die politische und kulturgeschichtliche Stoßrichtung, die der Verfasser bei dieser Ordnung der literarhistorischen Dinge verfolgt, lässt sich an seiner Darstellung Pindars besonders gut beobachten. Denn dessen Affinität zu den Gegnern der Griechen in den Perserkriegen erklärt er aus seiner „Abneigung gegen die Volksherrschaft“ und der „Vorliebe für die königliche Gewalt“. „Diese Form der Verfassung aber“, so heißt es bei Schlegel weiter, „die Monarchie und die Hoheit des Adels, erschien im Altertum [...] nirgends in einem so glänzenden und so milden Lichte, als in dem persischen Kaisertum“ (31). Für seine Einschätzung der pindarischen Oden bleibt der unterstellte Monarchismus ihres Dichters nicht ohne Folgen. Letztlich seien die Siegeslieder Pindars nämlich keine rätselhaften lyrischen Texte, sondern „[h]eroische oder epische Gelegenheitsgedichte [.. .], welche von Musik und Tanz begleitet, nicht bloß abgesungen, sondern gewissermaßen dramatisch aufgeführt wurden“ (32). Die kaiserliche Gesinnung des Dichters schließt Schlegel hier also sowohl mit dem Heldenepos - dem aus seiner Sicht prädestinierten Speicher aller Nationalerinnerungen - als auch mit einer öffentlichkeitswirksamen Dramatik kurz und schafft damit ein gattungspolitisches Dispositiv, das seine gesamte Literaturgeschichte durchzieht.

Allerdings basiert die historiographische Konzeption der Geschichte der alten und neuen Literatur auf der Grundannahme, dass die griechisch-römische Geisteskultur und die hebräisch-christliche kategorial verschieden seien (96). Für die Ausfaltung einer christlich-katholischen Genealogie der europäischen Literatur benötigt Schlegel also einen Fußpunkt jenseits der klassischen Antike. Und den setzt er mit den Hebräern. In unverkennbarem Anschluss an Herder, ${ }^{31}$ der durchweg als wichtiger Gewährsmann fungiert, weist Schlegel die Hebräer als dasjenige Volk aus, dessen „heilige Schriften [...] zugleich den Inbegriff [seiner] Literatur und Dichtkunst ausmachen“. ${ }^{32}$ In Gestalt der „Mosaischen Urkunde“ sowie in den „Gesängen Davids, den Sinnbildern Salomons, und den Weissagungen Jesajas“ hätten die Hebräer die „ihnen anvertraute Wahrheit und höhere Erkenntnis“ dann „rein und unverfälscht [.. .] auf die Nachwelt gebracht und erhalten“, ${ }^{33}$ und dies nicht zuletzt dank ihres ausgeprägten Originalgeistes, der sich - ähnlich dem der Griechen - gegen äußere Einflüsse abgeschlossen habe.

Das Alte Testament in seiner Ursprache so stark zu akzentuieren und es noch dazu als hebräische Dichtung zu lesen, schreibt sich nun aber unübersehbar aus

31 Herder 1993.

32 Ebd.: 146.

33 Ebd.: $100 \mathrm{f}$. 
dem Protestantismus und seiner exegetischen Tradition her. ${ }^{34}$ Und tatsächlich dürfte sich das Wiener Publikum verständnislos bis irritiert angesichts dieser Idee gezeigt haben, die im katholischen Habsburg keinerlei theologisch-philologische Fundierung besaß, geschweige denn publizistische Präsenz. Doch Schlegel versteht es, diesem entschieden protestantischen Ausgangspunkt einen konfessionsund staatspolitischen Dreh zu verleihen, der ihn für sein Projekt dienstbar macht. Zunächst behauptet er eine innige Verwandtschaft der persischen Lichtreligion mit dem Monotheismus der Hebräer, ${ }^{35}$ in dessen Licht er auch „die Staatsverfassung des persischen Kaisertums“ (99) sieht. Der persische Monarch habe nämlich, und mit der folgenden Metapher greift Schlegel kalkuliert auf eine Wendung aus den alttestamentlichen Propheten zurück, „als Sonne der Gerechtigkeit ein sichtbares Abbild des höchsten Gottes und des ewigen Lichts“ (ebd.) ${ }^{36}$ sein sollen. Mit dieser Analogiebehauptung zwischen persischem und hebräischem Kultus ${ }^{37}$ ist also einmal mehr das Kaisertum als Herrschaftsmodell ins, jetzt allerdings genuin monotheistische, Spiel gebracht - eine Staatsform, die die Hebräer selbst nicht vorweisen können.

Gleichzeitig löst Schlegel mit dieser Suggestion einer kaiserlich-monotheistischen Synthese im frühen Altertum auf kulturgeschichtlicher Ebene das intrikate Problem, dass die spätere Einführung des Christentums als Staatsreligion im Römischen Reich gerade nicht aus dem römischen „Originalgeist“ erwachsen war, sondern auf äußere, in diesem Fall orientalische, Einflüsse zurückging, was der Verfasser - wie erwähnt - grundsätzlich als Ausweis nationaler Schwäche wertet. Die historisch prominenteste Verbindung von Kaisertum und Monotheismus in Rom ist somit als potentieller Fußpunkt der Schlegel'schen Literaturhistoriographie desavouiert und wird funktional ersetzt durch die Behauptung jener persisch-hebräischen Kultuskonvergenz, die das persische Großreich mitsamt seiner kaiserlichen Herrschaftsform unter monotheistisches Vorzeichen stellt.

34 Zur Geschichte der protestantischen Exegese des Alten Testaments vgl. grundlegend Sheehan 2005; zum engen Wechselverhältnis dieser Tradition mit der deutschen Literatur vgl. ebenso grundlegend Weidner 2011.

35 „Die Perser verehrten und erkannten denselben Gott des Lichts und der Wahrheit, wie die Hebräer“ (99).

36 Die Wendung stammt aus dem Propheten Malechai; der entsprechende Vers lautet in Luthers Übersetzung: „Euch aber, die ihr meinen Namen fürchtet, soll aufgehen die Sonne der Gerechtigkeit und Heil unter ihren Flügeln.“ (Mal 3,20) Das heute unter diesem Titel bekannte Kirchenlied datiert deutlich später als Schlegels Vorlesungen.

37 Historisch sichert Schlegel diese Nähe von hebräischem und persischem Kultus ab durch Verweis auf die biblische Überlieferung von der Befreiung des jüdischen Volkes aus der babylonischen Gefangenschaft durch den Perserkönig Cyrus (vgl. 99). 
Wie stark Schlegel indes seinen welthistorischen Stoff tatsächlich literaturgeschichtlich ordnet, zeigt sich an einer zweiten argumentativen Volte, in der er die historisch so neuralgische Verbindung des Römischen mit dem Christlichen durch eine originär literarische Kopplung des Hebräischen mit dem Römisch-Katholischen ersetzt und zugleich die Brücke zur Neuzeit schlägt.

Dazu definiert er zunächst das „römische“ in ein „lateinisches“ Christentum um und betont, dass mit der lateinischen Bibelübersetzung „eine ganz neue Epoche“ der „römischen Sprache“ begonnen habe, zusammen mit einem „Aufschwung [...] der Sprache und der Literatur“ insgesamt, und zwar gerade nicht in Form einer Innovation, sondern einer ausgewiesenen renovatio: Allem voran in der Übersetzung der Psalmen habe die Vulgata den „orientalischen Ausdruck“ der hebräischen Bibel derart formvollendet nachgebildet, dass „die lateinische Sprache nun für alle folgenden Zeiten“ dessen „Spuren [. . .] behielt“ (154). Denn hier habe letztlich - und die folgende Winckelmann-Allusion ist ebenfalls altertumspolitisch motiviert - eine lateinische „Annäherung zu der orientalischen Erhabenheit“ stattgefunden, die ,in den heiligen Schriften der Hebräer [...] durchgängig mit edler Einfalt gepaart“ gewesen sei (155).

Das in dieser Form orientalisch renovierte Lateinische weist Schlegel dann nicht allein als entscheidenden Konnex zwischen Altertum und Neuzeit aus, der überdies den welt- und kulturgeschichtlichen Hiat zwischen antiker Philosophie und Christentum überbrückt habe (90), sondern er schreibt dieser in der Spätantike „noch lebenden“ Sprache eben auch einen zentralen Einfluss auf „den poetischen Geist des Mittelalters“ zu, und zwar durch „die aus ihr entsprungenen romanischen Sprachen“ (154). Entsprechend groß ist der Raum, den die Darstellung der romanischen Literaturen innerhalb der Vorlesungen einnimmt - darunter neben der italienischen und spanischen Literatur auch die portugiesische und provenzalische. ${ }^{38}$

Selbstverständlich versäumt es Schlegel bei aller Apologie der literarischen Romania nicht, auch den nordischen Sprachen und Literaturen einen zentralen Stellenwert beizumessen, wozu er einmal mehr auf das Altertum zurückgeht. Bereits die römischen Historiographen hätten den nordischen Völkern nämlich nicht allein eine besondere Freiheitsliebe bescheinigt, sondern „[s]o früh als nur die Römer der germanischen Völker erwähnen, unterlassen sie auch fast nie, der besonderen Liebe derselben zur Poesie zu gedenken“ (158). Entsprechend sei - und hier spricht wieder Herder aus dem Hintergrund - der eigentliche „Geist der Poesie“ letztlich aus den „nordischen Quellen seiner Bildung“

38 Die neunte, elfte und zwölfte Vorlesung sind überwiegend der Literatur der Romania gewidmet. 
nach Europa gelangt, wo er dann die mittelalterliche „Ritterpoesie“ hervorgebracht habe (158f.).

Eine dieser noch immer sprudelnden Quellen macht Schlegel in der isländischen Edda aus, wo er die nordische Götterlehre auf gattungspolitisch höchst signifikante Weise realisiert sieht. Die nämlich bilde, im Unterschied zur Mythologie der Griechen, selbst bereits eine „höhere Einheit“, was genauso dramenpoetisch gemeint ist, wie es klingt. Denn „durch die letzte Katastrophe, auf die alles prophetisch hindeutet“, so heißt es bei Schlegel im Verweis auf die Ragnarök, erhalte die nordische Götterlehre tatsächlich ,einen vollkommenen Schluß [...]. Es ist das Ganze wie ein einziges Gedicht, ein [. . .] Trauerspiel.“ (165)

Wenn aber die nordische Mythologie als solche bereits die Form einer Tragödie besitzt, dann erübrigt sich das verzweifelte Ringen um eine eigene deutsche Tragödientradition, das seit Herders Von deutscher Art und Kunst ${ }^{39}$ die deutsche und zwar allein die deutsche, nicht die österreichische - Poetik und Geschichtsphilosophie bestimmt hat und in der Nachfolge von Hegels Ästhetik dann zu einer eigenen Tradition gerinnen sollte. ${ }^{40}$ Die von seinen deutschen Zeitgenossen etablierte Gattungsgenealogie aus der attischen Tragödie einerseits und Shakespeare andererseits schreibt Schlegel also ebenso wenig fort, wie er die damit stets einhergehende Frontstellung zur französischen tragédie classique verlängert. Fernab der im deutschen Gattungsdiskurs gängigen antifranzösischen Ressentiments begegnet er Racine und Corneille mit ausgesprochener Wertschätzung und bescheinigt dem „Trauerspiel der Franzosen“ insgesamt, „der glänzendste Teil ihrer poetischen Literatur“ zu sein (297-303, hier 299).

Überhaupt verwendet Schlegel viel Mühe darauf, die europäische Literaturgeschichte als Synthese von nordischer und romanischer Tradition zu schreiben, woher auch seine große Zuneigung zur provenzalischen Dichtung - dem Hort des Minnesangs (183-185) - rührt, deren Sprache er als lateinisch-germanische Mischform rubriziert (183).

Doch gerade dieser europäische Horizont erlaubt es ihm, einen besonders spitzen Stachel ins Fleisch des deutschen tragödienpoetischen Narratems zu treiben: Das seinerzeit wortreich beklagte Fehlen einer großen deutschen Tragödie ist für Schlegel nämlich keineswegs, wie dies nicht allein bei den zeitgenössischen Hegelianern anklingt, durch den Umstand der unvollendeten Nation bedingt, sondern durch die Schwäche des Staates. Denn in der Geschichte der

39 Herder 1991.

40 Vgl. dazu die noch immer unerreichte Anthologie von Klaus Hammer (1987); darin das Vorwort des Herausgebers: 9-24. 
Neuzeit, so argumentiert Schlegel nicht ohne historische Plausibilität, habe es große Tragödien eben ausschließlich in starken Monarchien gegeben: Calderón und Lope de Vega in der spanischen Monarchie, die tragédie classique unter Ludwig XIV. und Jonson, Marlowe und Shakespeare im elisabethanischen England. Entsprechend könne es nicht verwundern, dass aktuell weder die Deutschen noch die Italiener eine profilierte Gattungstradition besäßen (277-293). Friedrich der Große fällt als monarchischer Motor der deutschsprachigen dramatischen Literatur in der Logik der Vorlesungen freilich schon aufgrund seiner Frankophilie und der damit verbundenen Orientierung an äußeren Einflüssen aus (237).

Wenn Schlegel seine abschließende Darstellung der Gegenwartsliteratur also ausgerechnet mit dem österreichischen Dramatiker Heinrich Collin enden lässt (405), dessen Römertragödien - insbesondere der 1802 mit einer Ouvertüre von Ludwig van Beethoven am Wiener Burgtheater uraufgeführte Corio$l a n^{41}$ - auf den Bühnen des gesamten deutschsprachigen Raums gespielt und gefeiert wurden, ${ }^{42}$ dann zieht er damit die aktualisierende Konsequenz aus seinem gattungspolitischen Einsatz, die nur auf Habsburg zulaufen kann.

\section{Konfession, Literatur und Europa: Lösungsversuche}

Dieser genuin staats- und nationalpolitische Strang der Geschichte der alten und neuen Literatur wird von Schlegel nun eng mit dem besagten konfessionellen verbunden. Dabei zeigt sich schnell, dass sich die dezidiert katholische Ausrichtung seiner literarhistorischen Ordnung nicht einfach auf eine persönliche Marotte des Verfassers zurückführen lässt, sondern unmittelbar mit seinem Bestreben zusammenhängt, eine tatsächlich europäische Literaturgeschichte in welthistorischem Horizont zu schreiben anstelle einer bloß nationalen. Denn während sich für nationalliterarische Projekte „preußisch-kleindeutschen“ Zuschnitts die Reformation, zumal in Gestalt Luthers, als integrale Bezugsgröße bekanntermaßen hervorragend eignet, markiert sie im europäischen Zusammenhang zunächst einmal das Moment einer weitreichenden Spaltung, das eben auch den deutschsprachigen Raum teilt. Dieses Moment spielt Schlegel

41 Collin 1804.

42 Zur Bedeutung Collins für die Konturierung der „Römertragödie“ des 19. Jahrhunderts vgl. Reimers 2016: 40-58. 
während der zweiten Hälfte seiner Vorlesungen immer wieder ein, ${ }^{43}$ verteilt diese Hinweise aber auf die Darstellung der einzelnen Nationalliteraturen und verschiebt seine Auseinandersetzung mit der Bedeutung Luthers für die deutschsprachige Literatur - in klarem Verstoß gegen die eigene chronologische Ordnung - auf die vorletzte Vorlesung, der er noch dazu den Gestus eines „Rückblick[s]“ verleiht (528).

Die deutsche Bibelübersetzung markiert für Schlegel freilich einen höchst neuralgischen Punkt, ruht die Gesamtanlage seiner Literaturgeschichte doch auf der unauflöslichen Verbindung von Nationalliteratur und Muttersprache auf, was eine Würdigung der Biblia deudsch, der protestantischen Umstellung des Gottesdienstes auf die Volkssprache sowie des lutherischen Liedguts als Dynamisierungsfaktoren der deutschsprachigen Literatur eigentlich unausweichlich machen müsste.

Doch Schlegel weiß Rat und legt den Grundstein seiner katholischen Gegenstrategie schon in der Spätantike, namentlich mit der bereits erwähnten Profilierung der Vulgata als kongenialer Übernahme des orientalischen Ausdrucks der alttestamentlichen Texte in die lateinische Sprache (150-161), die fortan als „Band“ sowohl zwischen Orient und Okzident als auch zwischen Antike und Neuzeit gedient und überdies während des gesamten Mittelalters die Einheit der europäischen Literatur garantiert habe, wenngleich deren poetischer Teil volkssprachig gewesen sei (171-173). Und selbst diese volkssprachige Poesie versteht Schlegel mit einer altkirchlichen Klammer zu versehen, die eine besondere semiotische bzw. tropische Gestalt besitzt und mit der spezifischen Poetik der Geschichte der alten und neuen Literatur eng verzahnt ist.

Die gesamten Vorlesungen ventilieren nämlich ein Verständnis von Dichtung als eo ipso allegorisch-sinnbildlicher Kunst. ${ }^{44}$ Schon in den biblischen Texten und den homerischen Epen sieht Schlegel einen „symbolischen Geist, und den daher erzeugten Hang zur Allegorie“ walten (212), ${ }^{45}$ der die mittelalterliche Dichtung in Europa dann durchweg und wesentlich bestimmt habe: vom Minnesang und den „Rittergedichte[n]“ (192) insgesamt, ganz besonders ausgeprägt bei Wolfram von Eschenbach (201), bis zu Dante (214ff.) und Calderón (286). Seinen altkirchlichen Anstrich gewinnt dieses Sinnbildlichkeitspostulat durch die zentrale Rolle, die Schlegel in diesem Zusammenhang der gotischen Baukunst zuweist, die er als vollkommen „[s]ymbolisch“ bestimmt, in ihren wiederkehrenden Grundformen des Kreuzes, der Rose und der Dreizahl als genuin

43 Vgl. 239-244, 253-260, 307-311 u.ö.

44 Ausführlich diskutiert findet sich diese Dimension der Vorlesungen in Brauers 1996: 312ff., $330 \mathrm{ff}$.

$45 \mathrm{Zu}$ Homer vgl. 44-48. 
„bedeutend und sinnbildlich“ ausweist (202-204) und über eben diese Eigenschaft mit der Dichtung des Mittelalters parallelisiert (202).

Tatsächlich laufen in Schlegels Apologie des Sinnbildlichen das konfessionspolitische, das nationalliterarische und das poetologische Kalkül der Vorlesungen zusammen: Da das sinnbildlich-allegorische Prinzip nicht nur die Differenz zwischen den Nationalsprachen übersteigt, sondern sogar transmedial wirksam ist, kann Schlegel es als ästhetischen Einheitsgaranten eines Europas setzen, dessen Dichtung von der Antike bis in die Gegenwart denselben Formgesetzen folgt, die allerdings in der mittelalterlichen Sakralkultur ihre reinste Ausprägung erfahren haben. Und im Horizont eben dieser langwelligen Kontinuität des Sinnbildlichen mit seiner translingualen und transmedialen Reichweite gelingt es Schlegel schließlich, die produktiven Auswirkungen der Reformation auf die deutsche Sprache als Literatursprache in einen kategorialen Bruch umzudefinieren:

„Das eigentliche Erwachen und Aufblühen der deutschen Sprache und alten Poesie“, so steht in der achten Vorlesung zu lesen, „beginnt mit Kaiser Friedrich dem Ersten im zwölften Jahrhundert.“ Zwar habe, anders als die zunehmend besser ausgebildete Prosa, die Poesie im Laufe der folgenden Jahrhunderte „zu verwildern“ begonnen, doch „Anfang des sechzehnten Jahrhunderts“ sei dann „mit einer allgemeinen Erschütterung der Begriffe, auch die gänzliche Veränderung mit der Sprache vor[gegangen], die nun eine Art Scheidewand zwischen uns und jener ältern deutschen Art und Kunst auch in der Sprache und Dichtung bildet“ (198). Schlegel deutet hier also - einmal mehr unter wirkmächtiger Einspielung Herders - die konfessionelle Ruptur der Reformation als endgültige Kappung des in doppeltem Wortsinn begrifflichen Bandes zwischen Mittelalter und Neuzeit und als Errichtung einer zumal literaturgeschichtlich höchst schmerzlichen „Scheidewand“. ${ }^{46}$

Damit hat sich Schlegel selbst den Weg geebnet, um Luthers Bibelübersetzung in ihrer Bedeutung für die Entwicklung der deutschen Hochsprache letztlich doch Tribut $\mathrm{zu}$ zollen, ohne gleichzeitig der Reformation das Wort zu reden. Um diese Gefahr endgültig zu bannen, wird er dabei nicht müde zu betonen, dass Luther mit seiner Übertragung des Bibeltextes letztlich nur an einigen wenigen Stellen von den „alten Lehrer[n] der Kirche“ (361) abgewichen, also größtenteils den Kirchenvätern gefolgt sei, was insofern nicht wunder nehmen könne, als die Biblia deudsch „bekanntlich nur durch Auswahl des Besten aus so vielen schon vor ihm vorhandenen Übersetzungen entstanden“ (362) sei.

46 Die explizite Auseinandersetzung mit Luther kurz vor Ende der Vorlesungen muss entsprechend auch nicht mehr sonderlich ausführlich ausfallen (vgl. $363 \mathrm{f}$.). 
Dem Sprachschöpfer Luther selbst schreibt Schlegel eine „große und starke Art des deutschen Ausdrucks“ (ebd.) zu, die er als „Volksschriftsteller“ entwickelt habe, von denen „kein anderes Land“ derart „merkwürdige, umfassende, vielwirkende“ besessen habe wie Deutschland (363f.). Literarisch wird Luther auf diese Weise also ins Kollektiv der Nation eingerückt und an die katholische Lehre zurückgebunden, wodurch sich auch die von der Reformation errichtete „Scheidewand“ zumindest ein wenig abschwächt.

Doch die eigentliche Kontinuität zwischen „uns“ und der „ältern deutschen Art und Kunst“ wird für Schlegel durch das Sinnbildliche garantiert, zu dem freilich - weil im Mittelalter zur höchsten Blüte gereift - die Dichter der katholischen Völker ungleich leichteren Zugang hatten und haben als die der protestantischen. So heißt es etwa zu Milton ${ }^{47}$ angesichts seines Paradise Lost:

\begin{abstract}
Gegen die katholischen Dichter, Dante und Tasso, die seine Vorbilder waren, stand er als Protestant auch dadurch im Nachteil, daß er von so manchen sinnbildlichen Vorstellungsarten, Geschichten und Überlieferungen, die jenen für ihre Poesie zum reichen Schmuck zu Gebote standen, keinen Gebrauch machen konnte. [.. .] Der Wert dieses epischen Werks liegt daher nicht sowohl in dem Plan des Ganzen, als in einzelnen Schönheiten und Stellen [...]. (295)
\end{abstract}

Wie sich in diesem Zitat bereits andeutet, hat die Setzung des Sinnbildlichen als Garant literaturgeschichtlicher Kontinuität auch Konsequenzen für die Hermeneutik. Denn obwohl sich Schlegel an keiner Stelle der Vorlesungen explizit gegen die (freilich durch und durch protestantisch codierte) historisch-kritische Methode wendet, läuft diese Bestimmung der Dichtung als sinnbildliche Kunst doch notwendig auf eine Apologie der (gleichfalls durch und durch katholisch codierten) Allegorese als dem einzigen Deutungsverfahren zu, das die tradierten Texte zu entschlüsseln und ihnen vor allem ihre überzeitliche Bedeutung zu entlocken vermag.

Im Bereich der Poesie selbst zieht Schlegel allerdings ganz explizite Konsequenzen aus diesem Dichtungsverständnis, indem er den poetischen Teil der Literatur unmissverständlich auf das Prinzip der uneigentlichen, ,indirekte[n] Darstellung“ verpflichtet und dies folgendermaßen begründet:

Die übersinnliche Welt, die Gottheit, und die reinen Geister können im ganzen nicht geradezu dargestellt werden; die Natur und die Menschheit sind die eigentlichen und nächsten Gegenstände der Poesie. Aber jene höhere und geistige Welt kann überall in diesen

47 Da Schlegel die anglikanische Kirche - mit Blick auf die Liturgik nicht ohne Plausibilität der katholischen zuschlägt (290), trifft der Vorwurf mangelnder Empfänglichkeit für die sinnbildlich-allegorische Tradition unter den englischen Dichtern die elisabethanischen - und damit auch Shakespeare - nicht, die Puritaner dafür aber umso mehr. 
irdischen Stoff eingehüllt sein, und aus ihm hervorschimmern. Ebenso ist auch die indirekte Darstellung der Wirklichkeit und Gegenwart, die beste und angemessenste. (276)

Der eigentliche Gegenstand der poetischen Darstellung ist, daran lässt Schlegel keinen Zweifel, „nur das Ewige, das immer und überall Bedeutende und Schöne“. Da sich dies allerdings nicht unmittelbar und „ganz ohne Hülle“ zur Darstellung bringen lässt, „,bedarf [die Poesie] dazu eines körperlichen Bodens, und diesen findet sie in ihrer eigenen Sphäre, der Sage oder der nationalen Erinnerung und Vergangenheit“" (ebd.).

Und hier schließt sich der Kreis: Die eingangs erwähnte nationalkonstitutive Hauptaufgabe der Dichtung, als Speicher der vaterländischen Erinnerungen zu fungieren, wird selbst unter das Vorzeichen des Sinnbildlichen gestellt, insofern die poetische Darstellung von Mensch, Leben und Welt dergestalt eingerichtet werden soll, dass sich stets „eine höhere Verklärung aller Dinge in ihrem Zauberspiegel ahnen läßt“ (ebd.). Dieses Verfahren nennt Schlegel „romantisch“ und rechnet entsprechend nicht allein die homerischen Epen dazu, sondern ,alles, was in indischen, persischen und andern alten orientalischen oder europäischen Gedichten wahrhaft poetisch ist“ (285f.). Im selben Zug justiert er auch die Frontlinien der Querelle des Anciens et des Modernes neu und hält fest:

Nicht dem Alten und wahrhaft Antiken, sondern nur dem unter uns fälschlich wieder aufgestellten Antikischen allein, was ohne innre Liebe bloß die Form der Alten nachkünstelt, ist das Romantische entgegengesetzt; so wie auf der andern Seite dem Modernen, d.h. demjenigen, was die Wirkung auf das Leben fälschlich dadurch zu erreichen sucht, daß es sich ganz an die Gegenwart anschließt, und in die Wirklichkeit einengt, wodurch es denn [...] der Herrschaft der beschränkten Zeit und Mode unvermeidlich anheim fällt. (286)

Allein eine in diesem Verständnis romantische Dichtung, die auf dem Wege der Sinnbildlichkeit ihren historischen Stoff mit dem Aufschein der „höhere[n] und geistige[n] Welt“ versieht, kann im Medium der Muttersprache über alle konfessionellen Differenzen und historische Distanzen hinweg die Nation mitformatieren.

Seit der Zersplitterung Deutschlands durch Reformation und Dreißigjährigen Krieg sowie der literarischen „Epoche der Barbarei“ zwischen Westfälischem Frieden und der Mitte des 18. Jahrhunderts ${ }^{48}$ sei, da ist sich Schlegel sicher, eine solche Literatur nun erstmals wieder im Aufgang begriffen. Begünstigt worden sei dies zweifellos durch das staatspolitische Wiedererstarken Österreichs, durch die Besteigung „mehrere[r] der ersten Throne[ ] in Europa von deutschen Fürs-

48 „[E]ine Art von Zwischenreich und chaotischem Mittelzustand in der deutschen Literatur, wo die Sprache selbst zwischen einem seinsollenden Halbfranzösisch, und einem verwilderten Deutsch schwankend, zugleich verkünstelt war und doch gemein“ (367). 
tenhäusern“ und den Aufstieg von „eine[m] derselben“ - der Verfasser hütet sich, Preußen beim Namen zu nennen - „zur königlichen Würde“ (367).

Doch literaturgeschichtlich lässt Schlegel die neue deutsche Poesie letztlich mit einem emergenten Ereignis beginnen, namentlich mit dem Erscheinen Klopstocks, der „ganz einsam, und fast allein damals in der deutschen Welt mit seinem hohen Nationalgefühl“ gewirkt habe, „welches nur von wenigen mitempfunden, von niemandem verstanden ward“ (369).

Der für eine fruchtbare Nationalliteratur unverzichtbaren Verschränkung von Sinnbildlichkeit und historischem Grund eingedenk, kann die herausgehobene Bedeutung von Klopstocks Epen und Dramen ${ }^{49}$ für die neue Blüte der deutschen Poesie ebenso wenig überraschen wie der Umstand, dass Schlegel weder dem bürgerlichen Trauerspiel noch dem Roman einen Anteil an diesem „Aufschwung der neueren deutschen Literatur“ (369) zubilligt: Lessings Trauerspiele seien schließlich nichts weiter als „Familiengemälde in Prosa“ (384), die dank Shakespeare dramengeschichtlich Episode geblieben seien. Und der Roman bleibe - von Cervantes abgesehen - so sehr in der Beschränktheit des Wirklichen befangen, dass ihm als Mittel zur vermeintlich poetischen Übersteigung desselben nur Fernreisen der Protagonisten zu Gebote stünden, oder der Rückgriff auf das, was Schlegel nicht ohne Sinn für Pointen das „Polizeiwidrige“ (275) nennt. $^{50}$

Und so teilt Schlegel zum Abschluss seiner Vorlesungen die eigene Gegenwartsliteratur - methodisch durchaus richtungsweisend - in drei „Generationen“ ein: ${ }^{51}$ Die erste umfasst neben Klopstock auch Gleim, Johann Elias Schlegel, Ewald von Kleist, Haller, Uz, Wieland und den österreichischen Jesuitendichter Michael Denis, unter den „Prosaisten“ Lessing, Mendelssohn, Sulzer und Winckelmann (377f.). In die zweite rechnet Schlegel den jungen Goethe sowie Stolberg, Bürger, Voß, Jacobi, Lavater, Herder und - für sein Konzept einer deutschen Nationalliteratur bezeichnend - den Schweizer Johannes Müller

49 Diese Stellung bei Schlegel verdankt Klopstock einzig seiner christlich-nordischen Stoffwahl. In seiner poetischen Gestaltung habe Klopstock dagegen zu stark auf fremde Formen zurückgegriffen, den Antiken nachgekünstelt und außerdem den in der deutschen Dichtung so tief verankerten Reim abgeschafft, vgl. 372-374.

50 Darunter subsumiert Schlegel Verstöße der Protagonisten gegen die rechtliche, öffentliche oder sittliche Ordnung, die er als sine qua non des Romans mit seinem in jeder Hinsicht prosaischen Gegenwarts- und Wirklichkeitsbezug ausmacht. Ohne dass hier Namen oder Titel genannt würden, lassen sich in Schlegels exempla-Reihe mühelos die großen englischen Romane des 18. Jahrhunderts, der Wilhelm Meister und Schlegels eigene Lucinde erkennen.

51 Zur Begründung dieser Einteilung vgl. 377. 
(381-383). ${ }^{52}$ Und zur dritten Generation zählt er unter den Philosophen Kant, Fichte, Schelling, unter den Dichtern Schiller, Jean Paul, den mittleren Goethe, Novalis und schließlich den bereits erwähnten Heinrich Joseph Collin (392-410).

Charakteristisch für diese dritte Generation, deren Angehörige Schlegel von Georg Forster abgesehen - keineswegs als tatsächliche Revolutionäre verhandelt, sei ihre „revolutionäre Epoche“ gewesen (393), die alle Literaten dazu genötigt habe, sich auf die eine oder andere Weise intensiv mit ihrem Zeitalter auseinanderzusetzen (394) und dabei einen beispiellosen „Ideenreichtum“ zu entwickeln. Entsprechend hält der Verfasser auch mit Blick auf sich selber fest: „[W]essen Bildung und Entwicklung in diese Zeit von 1788-1802 fiel, der wird sie ungeachtet jener nachteiligen Verhältnisse nicht leicht aufgeben, oder mit einer andern vertauschen wollen.“ (396f.)

So ist es für die literatur- und nationalpolitische Programmatik der Vorlesungen nicht ohne Signifikanz, dass Schlegel abschließend anmerkt, er „sehe [...] eine neue Generation entstehen und sich bilden und ohne Zweifel wird das neunzehnte Jahrhundert auch in unserer Literatur sich ganz anders gestalten als das achtzehnte war“ (406). Und weiter heißt es:

Wenn von dem Ganzen der deutschen Literatur die Rede ist, so zweifle ich auch keinen Augenblick daran, daß sie noch alle die großen Erwartungen erfüllen wird, welche sie bisher mehr nur lebhaft angeregt hat, als vollständig zu befriedigen vermochte. (407)

Jenes „Ganze[ ] der deutschen Literatur“ historiographisch zu formatieren und es zu nationalem (Selbst-)Bewusstsein zu bringen, hat Schlegel mit seiner $\mathrm{Ge}$ schichte der alten und neuen Literatur unternommen, deren weltliterarischer Horizont mit europäischem Fokus zugleich die Bedingung der Möglichkeit darstellt, die deutsche Sprache als "für den höheren wissenschaftlichen Gebrauch“ prädestinierte (232) und somit als ideales Medium der „Geschichte als Wissenschaft“ (337) nicht allein zu behaupten, sondern sie in diesem Vermögen auch selbst vorzuführen.

52 Dem Verfasser der Schweizer Geschichte (1786ff.), der seit 1792 in österreichischen Diensten stand, kommt für Schlegels Autorenauswahl eine wichtige Integrationsfunktion für die gesamtdeutsche Literatur zu. Seine alpine Herkunft wird an anderer Stelle sogar noch einmal eigens akzentuiert und als topographische Untersetzung politischer Weitsicht inszeniert. So heißt es von der zweiten Generation der neuen deutschen Literatur, ihre Angehörigen hätten „in einer uns fast sonderbar erscheinenden Sorglosigkeit“ gelebt und die „Symptome der herannahenden Gefahren und Erschütterungen“ verkannt. „Der einzige Johannes Müller macht hier eine Ausnahme, dessen Geist ganz auf diese Gegenstände gerichtet, von der einsamen Höhe seiner Alpen freilich die heraufziehenden Gewitterwolken früher und deutlicher erkennen mußte, als die unten im friedlichen Tal oder in dem Gewirre der Städte Wohnenden.“ (394) 
Dass Heinrich Heine mit seinem viel zitierten Diktum, der „hohe Standpunkt“, von dem der Verfasser hier die ganze Literatur überblicke, sei „doch immer der Glockenturm einer katholischen Kirche“, 53 den Zuschnitt der Schlegel'schen Historiographie sehr präzise beschreibt, hat sich im Verlauf dieses Beitrags noch einmal in aller Deutlichkeit abgezeichnet. Tatsächlich präsentiert sich der Katholizismus der Vorlesungen als konstitutives Moment einer Literaturgeschichtsschreibung, die auf eine nordisch-romanische Einheit Europas vor dem Traditionshintergrund des östlichen wie westlichen Altertums ebenso abzielt wie auf die performative Hervorbringung einer deutschen Nation, die den gesamten deutschsprachigen Raum umfasst und in der staatlichen Fassung des österreichischen Kaisertums gedacht ist.

Die Relevanz der Geschichte der alten und neuen Literatur für ein gegenwärtiges Nachdenken über das Deutsche als Wissenschaftssprache, das mit Friedrich Schlegel als integraler Bestandteil des Deutschen als Literatursprache verstanden sein will, liegt freilich nicht in ihrer aktuellen weltanschaulichen Affirmierbarkeit, sondern darin, dass diese Vorlesungen einen programmatischen Gegenentwurf zur zeitgenössischen „preußisch-kleindeutschen“ Ordnung der historischen und nationalen Dinge unternehmen, in dessen kontrastierendem Licht die Warte der heutigen deutsch(sprachig)en Literaturgeschichtsschreibung unverkennbar Konturen eines Bismarckturms aufweist.

Man mag die Aussicht auf die wissenschafts- und literatursprachliche Landschaft, die sich von diesem speziellen Bauwerk aus bietet, mit Fug derjenigen von einem katholischen Glockenturm vorziehen, und sei es auch nur aus Gewohnheit. Doch um der literaturgeschichtlichen und wissenschaftssprachlichen Sache willen dürfte sich ein Umzug an einen dritten Ort lohnen, wo immer er liegt. Denn womöglich ließe sich von ihm aus - frei von aller (Auto-) Suggestion einer alternativlosen Perspektive - das so herrlich intrikate Verhältnis von deutscher Sprache, deutschsprachiger Literatur und Wissenschaft, einer wie auch immer gearteten deutschen Nation und den an ihrer Formatierung mitwirkenden Staaten in Vergangenheit und Gegenwart tatsächlich verstehen und wissenschaftlich wie gesellschaftlich fruchtbar machen. Langeweile würde bei einer solchen Unternehmung sicherlich nicht aufkommen.

53 Heine 1979: 167. 


\section{Literatur}

Bauer, Manuel (2014): Konversionen in Friedrich Schlegels später Literaturkritik, Philologie und Hermeneutik. In: Winfried Eckel (Hrsg.), Figuren der Konversion. Friedrich Schlegels Übertritt zum Katholizismus im Kontext. Paderborn: Schöningh, 160-179.

Behler, Ernst (1996): Friedrich Schlegel. Mit Selbstzeugnissen und Bilddokumenten. Reinbek: Rowohlt.

Böhler, Michael (2002): Vom Umgang der Literaturwissenschaft mit kulturtopographischen Aspekten der deutschsprachigen Literatur. In: Ders. \& Hans Otto Horch (Hrsg.), Kulturtopographie deutschsprachiger Literaturen. Perspektivierungen im Spannungsfeld von Integration und Differenz. Tübingen: De Gruyter, 11-44.

Brauers, Claudia (1996): Perspektiven des Unendlichen. Friedrich Schlegels ästhetische Vermittlungstheorie. Die freie Religion der Kunst und ihre Umformung in eine Traditionsgeschichte der Kirche. Berlin: Erich Schmidt.

Breuer, Ulrich (2010): Friedrich Schlegel. In: Wolfgang Bunzel (Hrsg.), Romantik. Epoche Autoren - Werke. Darmstadt: WBG, 60-75.

Breuer, Ulrich (2017): Lebensstationen. In: Johannes Endres (Hrsg.), Friedrich Schlegel Handbuch. Leben - Werk - Wirkung. Stuttgart: J.B. Metzler, 29-32.

Busch, Christopher (2011): Kontinuität der Form? Zum Verhältnis von Philologie, Charakteristik und Literaturgeschichtsschreibung bei Friedrich Schlegel. In: Athenäum 21, 17-46.

Collin, Heinrich Joseph von (1804): Coriolan. Ein Trauerspiel in fünf Aufzügen. Berlin: Friedrich Unger.

Eichendorff, Joseph von (1980): Werke. Bd. 4: Nachlese der Gedichte. Erzählerische und dramatische Fragmente. Tagebücher 1798-1815. Hrsg. von Klaus-Dieter Krabiel \& Marlies Korfsmeyer. München: Winkler.

Eichner, Hans (1961): Einleitung. In: Kritische Friedrich-Schlegel-Ausgabe. Bd. 6. Hrsg. u. eingel. von Hans Eichner. München, Paderborn, Wien: Schöningh, XI-XLVII.

Endres, Johannes (2017): Über die Sprache und Weisheit der Indier. In: Ders. (Hrsg.), Friedrich Schlegel Handbuch. Leben - Werk - Wirkung. Stuttgart: J.B. Metzler, 218-224.

Fohrmann, Jürgen (1989): Das Projekt der deutschen Literaturgeschichte. Entstehung und Scheitern einer nationalen Poesiegeschichtsschreibung zwischen Humanismus und Deutschem Kaiserreich. Stuttgart: J.B. Metzler.

Hammer, Klaus (Hrsg.) (1987): Dramaturgische Schriften des 19. Jahrhunderts. Berlin: Henschelverlag.

Heine, Heinrich (1979): Die romantische Schule. In: Historisch-kritische Gesamtausgabe der Werke. Bd. 8.1. Bearb. von Manfred Windfuhr. Hamburg: Hoffmann und Campe, 121-243. Herder, Johann Gottfried (1991): Von deutscher Art und Kunst. In: Werke in zehn Bänden. Bd. 2: Schriften zur Ästhetik und Literatur 1767-1781. Hrsg. von Gunter E. Grimm. Frankfurt a.M.: Deutscher Klassiker Verlag, 498-521.

Herder, Johann Gottfried (1993): Vom Geist der Ebräischen Poesie. Eine Anleitung für die Liebhaber derselben und der ältesten Geschichte des menschlichen Geistes. In: Werke in zehn Bänden. Bd. 5: Schriften zum Alten Testament. Hrsg. von Rudolf Smend. Frankfurt a.M.: Deutscher Klassiker Verlag, 661-1301.

Herrmann, Britta (2015): Für eine wahrhaft deutsche Kunst und Rede. Friedrich Schlegel und Adam Müller. In: Athenäum 25, 227-248. 
Jaeger, Friedrich \& Jörn Rüsen (1992): Geschichte des Historismus. Eine Einführung. München: C.H. Beck.

Marquardt, Hans-Jochen (1995): Zur ästhetischen Theorie des deutschen Frühkonservatismus. Friedrich Schlegels und Adam Heinrich Müllers Wiener Vorlesungen von 1812. In: Acta Germanica 23, 21-39.

Matuschek, Stefan (2001): Poesie der Erinnerung. Friedrich Schlegels Wiener Literaturgeschichte. In: Günter Oesterle (Hrsg.), Erinnern und Vergessen in der europäischen Romantik. Würzburg: Königshausen \& Neumann, 193-205.

Michler, Werner (2011): Austrian Literature of the 18th Century. In: Thomas Wallnig, Johannes Frimmel \& Werner Telesko (Hrsg.), 18th Century Studies in Austria 1945-2010. Bochum: Dieter Winkler, 187-206.

Müller, Adam (1967): Zwölf Reden über die Beredsamkeit und deren Verfall in Deutschland. Gehalten zu Wien im Frühlinge 1812. In: Kritische, ästhetische und philosophische Schriften. Bd. 1. Hrsg. von Walter Schroeder \& Werner Siebert. Neuwied, Berlin: Luchterhand, 293-451.

o.A. (1812): Oesterreichischer Beobachter Nr. 50, 19. Februar 1812, Rubrik „Wissenschaftliche und Kunstnachrichten“, o.P.

Osinski, Jutta (1993): Katholizismus und deutsche Literatur im 19. Jahrhundert. München, Paderborn, Wien: Schöningh.

Polaschegg, Andrea (2017): Geschichte der alten und neuen Literatur. In: Johannes Endres (Hrsg.), Friedrich Schlegel Handbuch. Leben - Werk - Wirkung. Stuttgart: J.B. Metzler, 224-233.

Polaschegg, Andrea (2019): Der konvertierte Meilenstein. Friedrich Schlegels Geschichte der alten und neuen Literatur (1812/14). In: Publications of the English Goethe Society 88 (1), 39-53.

Pornschlegel, Clemens (2017): Allegorien des Unendlichen. Hyperchristen II. Studien zum religiösen Engagement in der literarischen Moderne. Kleist, Schlegel, Eichendorff, Hugo Ball. Berlin, Wien: Turia + Kant.

Reimers, Timm (2016): Gelehrsamkeit, Politik und Spektakel. Transformationen der deutschen Römertragödie 1800-1900. Berlin, Boston: De Gruyter, 40-58.

Schlegel, Friedrich (1811): Ueber die neuere Geschichte. Vorlesungen gehalten zu Wien im Jahre 1810 von Friedrich Schlegel. Wien: Schaumburg.

Schlegel, Friedrich (1815): Friedrich Schlegels Geschichte der alten und neuen Litteratur. Vorlesungen gehalten zu Wien im Jahre 1812. 2 Bde. Wien: Schaumburg.

Schlegel, Friedrich (1818): Lectures on the History of Literature, Ancient and Modern. From the German of Frederick Schlegel. 2 Bde. Edinburgh: W. Blackwood.

Schlegel, Friedrich (1841a): Friedrich von Schlegel's Geschichte der alten und neuen Literatur. Bis auf die neueste Zeit fortgeführt von Theodor Mundt. 2 Bde. Berlin: M. Simion.

Schlegel, Friedrich (1841b): Lectures on the History of Literature, Ancient and Modern. From the German of Frederick Schlegel. Übers. von John Gibson Lockhart. New York: Langley.

Schlegel, Friedrich (1859): Lectures on the History of Literature, Ancient and Modern. From the German of Frederick Schlegel. Now First Completely Translated and Accompanied by a General Index. London: Bohn.

Schlegel, Friedrich (1911): Geschichte der alten und neuen Literatur. Vorlesungen gehalten zu Wien im Jahre 1812. Hrsg. von Marie Speyer. Mit einem ergänzenden Schlußkapitel von Wilhelm Kosch. Regensburg: J. Habbel. 
Schlegel, Friedrich (1961): Geschichte der alten und neuen Literatur. In: Kritische FriedrichSchlegel-Ausgabe. Bd. 6. Hrsg. u. eingel. von Hans Eichner. München, Paderborn, Wien: Schöningh.

Schlegel, Friedrich (1975): Über die Sprache und Weisheit der Indier. In: Kritische FriedrichSchlegel-Ausgabe. Bd. 8: Studien zur Philosophie und Theologie [1796-1824]. Hrsg. von Ernst Behler. München, Paderborn, Wien: Schöningh, 105-433.

Schöning, Matthias (2017): Geschichte und Politik. In: Johannes Endres (Hrsg.), Friedrich Schlegel Handbuch. Leben - Werk - Wirkung. Stuttgart: J.B. Metzler, 238-263.

Sheehan, Jonathan (2005): The Enlightenment Bible. Translation, Scholarship, Culture. Princeton: Princeton University Press.

Wegmann, Nikolaus (2017): Schlegels intellektuelle Konversion. Eine Skizze. In: Winfried Eckel (Hrsg.), Figuren der Konversion. Friedrich Schlegels Übertritt zum Katholizismus im Kontext. Paderborn: Schöningh, 148-159.

Weidner, Daniel (2011): Bibel und Literatur um 1800. München: Wilhelm Fink.

Wellek, René (1978): Geschichte der Literaturkritik. Bd. 1: Das späte 18. Jahrhundert. Das Zeitalter der Romantik. Berlin, New York: De Gruyter.

Windisch, Ernst (1992): Geschichte der Sanskrit-Philologie und Indischen Altertumskunde. I., II. Teil sowie nachgelassene Kapitel des III. Teils. Um ein Namen- und Sachverzeichnis zum III. Teil erweiterter, ansonsten unveränderter Nachdruck der Ausgabe von 1917, 1920 und 1921. Berlin, New York: De Gruyter.

Zeyringer, Klaus \& Helmut Gollner (2012): Eine Literaturgeschichte. Österreich seit 1650. Innsbruck, Wien, Bozen: Studienverlag. 